\title{
Acute intraabdominal hemorrhage from an aneurysm on uterine artery
}

\author{
Tomoko Nakagawa ${ }^{1}$, Yutaka Kyukawa ${ }^{2}$, Yukiharu Nakaoka ${ }^{3}$, Teruhisa Ninoi ${ }^{4}$, Kenji \\ Nakamura $^{5}$, Yujiro Tsujita ${ }^{6}$, Ken-ichi Honda ${ }^{1 *}$, Yasushi Kurihara', Hiroyuki Terada ${ }^{1}$, Tetsuji \\ Ando $^{1}$, Tomoyo Yasui ${ }^{7}$, Toshiyuki Sumi ${ }^{7}$, Masayasu Koyama ${ }^{7}$
}

\begin{abstract}
${ }^{1}$ Department of Obstetrics and Gynecology, Kashiwara Municipal Hospital, 1-7-9, Hozenji, Kashiwara, Osaka, Japan ${ }^{2}$ Yutaka Maternity Ultrasound Clinic, 1-6-6, Uriwari, Hirano-ku, Osaka, Osaka, Japan

${ }^{3}$ Nakaoka Ladies Clinic, Hannancho, Abeno-ku, Osaka, Osaka, Japan

${ }^{4}$ Department of Radiology, PL Hospital, 2204, Shindo, Tondabayashi, Osaka, Japan

${ }^{5}$ Department of Radiology, Daito Chuou Hospital, 2-1-11, Ohno, Daito, Osaka, Japan

${ }^{6}$ Department of Radiology, Kashiwara Municipal Hospital, 1-7-9, Hozenji, Kashiwara, Osaka, Japan

${ }^{7}$ Department of Obstetrics and Gynecology, Osaka City Univ. Grad. Sch. Med, 1-4-3, Asahimachi, Abeno-ku, Osaka, Japan
\end{abstract}

Received: 06 January 2017

Revised: 25 February 2017

Accepted: 06 March 2017

\author{
*Correspondence: \\ Ken-ichi Honda, \\ E-mail: kenhonda@med.osaka-cu.ac.jp
}

Copyright: (c) the author(s), publisher and licensee Medip Academy. This is an open-access article distributed under the terms of the Creative Commons Attribution Non-Commercial License, which permits unrestricted non-commercial use, distribution, and reproduction in any medium, provided the original work is properly cited.

\begin{abstract}
A 36-year-old woman was underwent emergency laparotomy for acute intraabdominal hemorrhage, but bleeding points were not found. Abdominal pains continued after the laparotomy, and rupture of aneurysm on uterine artery was found in angiography. An transcatheter arterial embolization was done for the uterine artery, and the aneurysm was found to disappear in 4-day-after the angiography. Rupture of an aneurysm on uterine artery should be considered for the causes of acute intraabdominal hemorrhage.
\end{abstract}

Keywords: Aneurysm on uterine artery, Angiography, Arterial embolization, Intraabdominal hemorrhage

\section{INTRODUCTION}

As causes of intraabdominal hemorrhage, ovarian bleeding, rupture of ovarian cyst or ectopic pregnancy are commonly considered. Ruture of aneurysm on uterine artery was found by angiography and transcatheter embilization was done after laparotomy for massive hemorrhage in intraabdominal space. The patient recovered from pre-shock status without hemostasis during laparotomy, probably because of temporal covering over the ruptured aneurysm.

\section{CASE REPORT}

A 36-year-old woman, gravida 0, visited department of internal medicine in our hospital for right lower abdominal pain continuing for 2 days. Laboratory data showed mild anemia ( $\mathrm{Hb} 10.6 \mathrm{~g} / \mathrm{dL})$, and swelling of right ovary was found in CT (Figure 1). On the 3rd day after the visit, the patient was introduced to our department, and swollen right ovary $(3.9 \times 2.8 \mathrm{~cm})$ and a submucous myoma $(3.3 \times 3.0 \mathrm{~cm})$ in uterus was found in ultrasound examination. On the 4th day after the visit, the patient was transferred to our hospital for strong abdominal pains. Transvaginal ultrasound examination revealed echo free space in Dougal pouch and vesicouterine fossa, suggesting intraabdominal hemorrhage. The pregnancy test was negative, ovarian bleeding was most likely suspected. The patient showed pre-shock status; arterial blood pressure $89 / 58 \mathrm{mmHg}$, heart rate $100 /$ min, and laboratory test showed anemia ( $\mathrm{Hb} 7.8$ $\mathrm{g} / \mathrm{dL}$ ). An emergency laparotomy was done under blood 
transfusion of 6 units of MAP, and about $1000 \mathrm{~mL}$ blood pooling was found in abdominal cavity. However, no bleeding point was found on bilateral adnexa or on uterus.

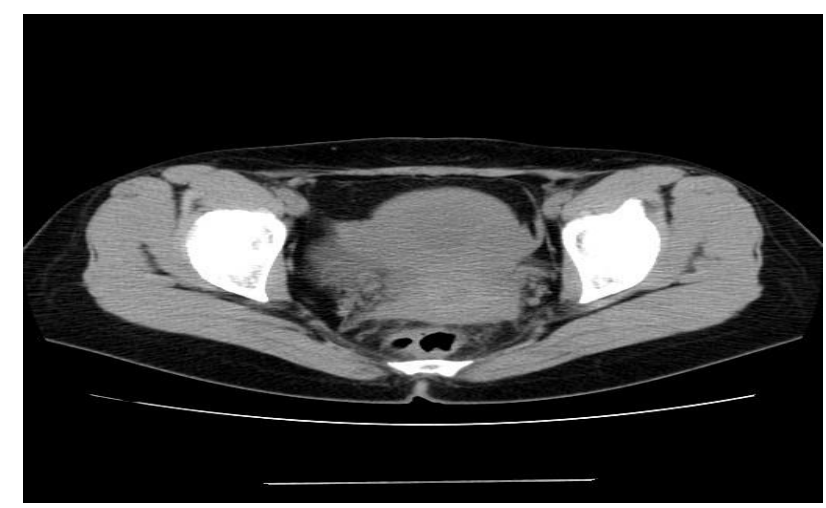

Figure 1: CT of pelvic cavity before angiography of pelvic cavity. Swelling of right ovary was observed.

General status recovered soon after the laparotomy, but pain continued in right lower abdomen. Enhanced CT with contrast medium revealed an aneurysm-like arterial blood pooling on right uterine artery (Figure 2 Figure 3 ).

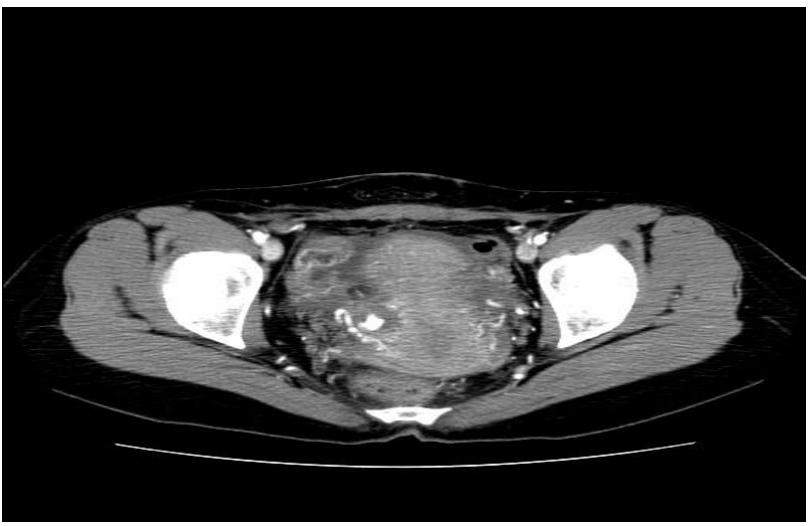

Figure 2: Enhanced CT of pelvic cavity after emergency laparotomy. An aneurysm-like blood pooling was found on right uterine artery.

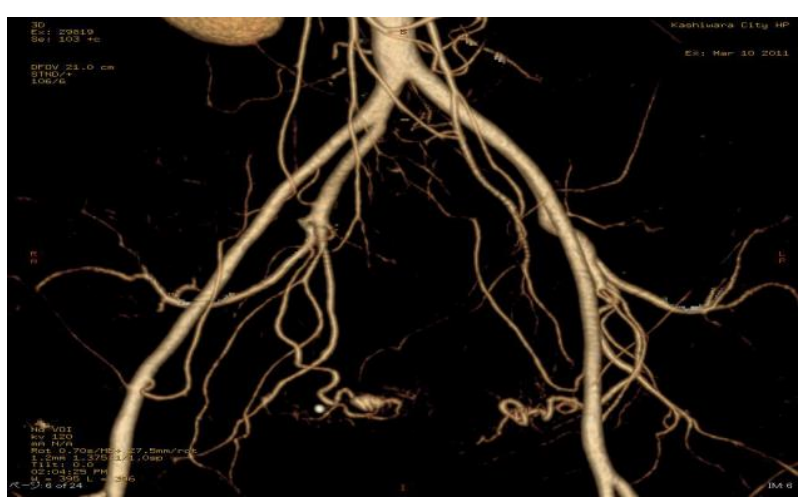

Figure 3: CT angiogram of pelvic cavity after emergency laparotomy. An aneurysm-like blood pooling was found on right uterine artery.
Transcatheter embolization of right uterine artery was done with gelatin sponge particles as temporary embolic materials (Figure 4) and a metallic coil in proximal portion (Figure 5, 6). The aneurysm was found to be disappeared (Figure 5,6). The patient was saying right lower abdominal pain disappeared after the transcatheter embolization.

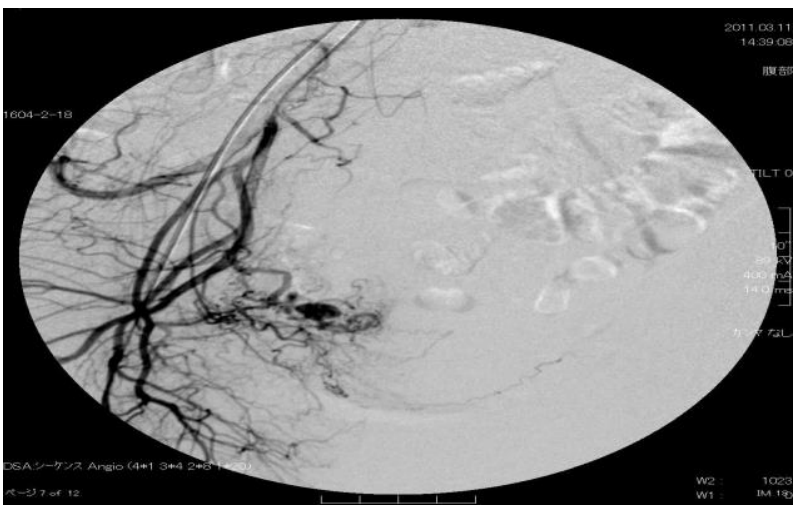

Figure 4: Angiography of pelvic cavity before embolization of right uterine artery. Leakage from pseudo-aneurysm was found on right uterine artery.

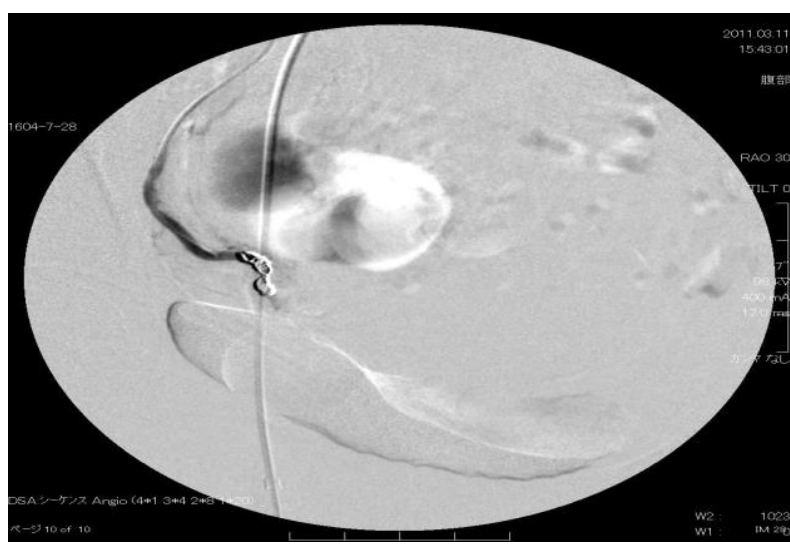

Figure 5: Angiography of pelvic cavity after embolization of right uterine artery. No leakage was found on right uterine artery.

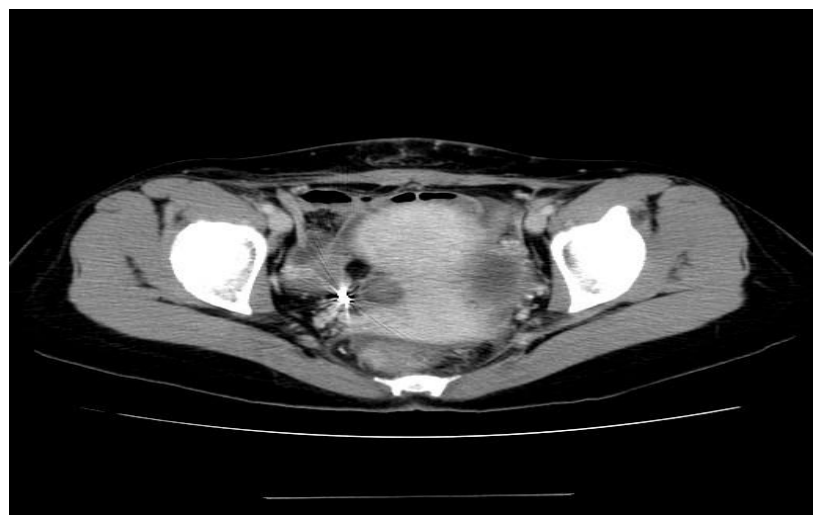

Figure 6: Enhanced CT of pelvic cavity after emergency laparotomy. No leakage was found on right uterine artery. 


\section{DISCUSSION}

From the angiography, the cause of intraabdominal hemorrhage of the patient seemed to be rupture of the aneurysm of uterine artery. During laparotomy, bleeding from uterine artery might be suppressed by pseudoaneurysm with ruptured vessel and other materials. Pains continued because of small amount of blood leak from injured vessel walls, and embolization of uterine artery blocked the blood leakage.

As causs of aneurysm, Marfan syndrome and LoeysDietz syndrome are possible diseases in which connective tissues lose the elasticity. ${ }^{1}$ Other characteristics of these diseases like high statue, long extremities, and long fingers were not found in this patient.

True aneurysm has a three-layered arterial wall. Dysfunction of vascular smooth muscle cells and inappropriate activation of proteolytic enzymes are studied as related to pathogenesis of aneurysm, and a serine proteinase inhibitor, kallistatin, is proposed as a protective drug for abdominal aortic aneurysm. ${ }^{1}$

There are reports of aneurysm complicated with pregnant women. It is hypothesized that increased levels of estrogen and progesterone lead to degeneration of arterial wall aneurysms. ${ }^{2}$ Weakness of arterial wall and increased blood volume may also predispose for the aneurysm. Splenic artery aneurysm is rare, but rupture events occur usually in pregnant women. ${ }^{2}$

Bacterial or fungal inflammation of the vessel wall can lead to dilatation of the vessel and formation of aneurysm. ${ }^{3}$

Pseudoaneurysm that doesn't have three arterial layers is rather more often reported as causes of rupture events from uterine artery than true aneurysm. Pseudoaneurysms are often related to surgical injury to uterus, cesarean section, curettage, vaginal delivery, or conization. ${ }^{4,5}$

Rupture of an aneurysm on uterine artery should be included in the diseases differentiated for the cause of acute intraadominal hemorrhage, especially in gynecologic patients.

\section{CONCLUSION}

Aneurysm and temporal hemostasis on uterine artery was found by angiography and transcatheter embolization was done after laparotomy for massive intraabdominal hemorrhage. Rupture of aneurysm on uterine artery should be considered as a cause of sudden intraabdominal hemorrhage.

Funding: No funding sources

Conflict of interest: None declared

Ethical approval: Not required

\section{REFERENCES}

1. Li J, Krishna SM, Golledge J. The potential role of Kallistatin in the development of abdominal aortic aneurysm. Int J Mol Sci. 2016;17:1312.

2. Heitkamp AC, Dickhoff C, Nederhoed JH, Franschman G, de Vries JI. Saved from a fatal flight: A ruptured splenic artery aneurysm in a pregnant woman. Int J Surg Case Rep. 2015;8:32-4.

3. van Doom DEA, van Leuken M, Rijnders RJP. Embolization of a left uterine artery mycotic aneurysm after a neglected, perforated appemdicitis before delivery. Clinical Case Reports. 2014;2:18890.

4. Chummum K, Kroon N, Flannelly G, Brophy D. Severe postcoital bleeding from a uterine artery pseudoaneurysm 4 months after cesarean section. Obstet Gynecol. 2015;126:638-41.

5. Moon G, Joen S, Nam K-H, Choi S, Sunwoo J, Ryu A. Pseudoaneurysm of uterine artery causing intraabdominal and vaginal bleeding after cervical conization. Obstet Gynecol Sci. 2015;58:256-9.

Cite this article as: Nakagawa T, Kyukawa $Y$, Nakaoka Y, Ninoi T, Nakamura K, Tsujita Y, et al. Acute intraabdominal hemorrhage from an aneurysm on uterine artery. Int J Reprod Contracept Obstet Gynecol 2017;6:1646-8. 